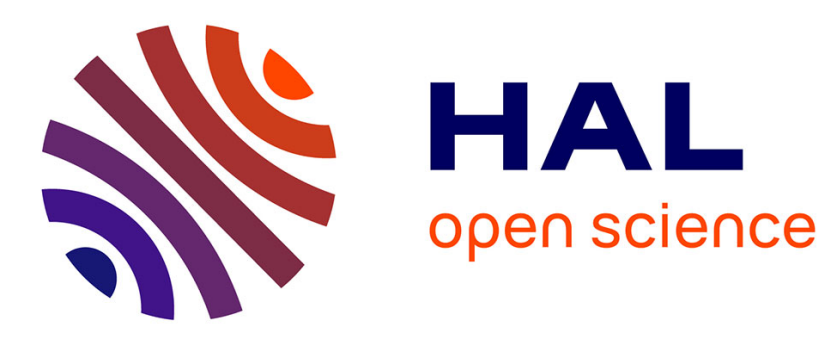

\title{
SEU mechanisms in spintronic devices: Critical parameters and basic effects
}

\author{
Odilia Coi, Nomena Adrianjohany, Gregory Di Pendina, David Dangla, \\ Robert Ecoffet, Bernard Diény, Lionel Torres
}

\section{- To cite this version:}

Odilia Coi, Nomena Adrianjohany, Gregory Di Pendina, David Dangla, Robert Ecoffet, et al.. SEU mechanisms in spintronic devices: Critical parameters and basic effects. IEEE Transactions on Nuclear Science, 2021, 68 (8), pp.1533-1541. 10.1109/TNS.2021.3080080 . hal-03252884

\section{HAL Id: hal-03252884 \\ https://hal.science/hal-03252884}

Submitted on 8 Jun 2021

HAL is a multi-disciplinary open access archive for the deposit and dissemination of scientific research documents, whether they are published or not. The documents may come from teaching and research institutions in France or abroad, or from public or private research centers.
L'archive ouverte pluridisciplinaire HAL, est destinée au dépôt et à la diffusion de documents scientifiques de niveau recherche, publiés ou non, émanant des établissements d'enseignement et de recherche français ou étrangers, des laboratoires publics ou privés. 


\title{
SEU Mechanisms In Spintronic Devices: Critical Parameters and Basic Effects
}

\author{
Odilia Coi*†‡, Nomena Andrianjohany ${ }^{\S}$, Gregory Di Pendina*, Lionel Torres ${ }^{\dagger}$, David Dangla ${ }^{\ddagger}$, \\ Bernard Dieny * and Robert Ecoffet ${ }^{\ddagger}$ \\ *SPINTEC Univ. Grenoble Alpes/CNRS/CEA IRIG/CEA 38054 Grenoble, France \\ ${ }^{\dagger}$ LIRMM-University of Montpellier, CNRS, France \\ ${ }^{\ddagger}$ Centre National d’Études Spatiales (CNES) 31401 Toulouse, France \\ $\S$ TRAD Test \& Radiations, 31670 Labège, France
}

\begin{abstract}
The paper investigates radiation-induced switching mechanisms, temperature effects, breakdown voltage, sensitive volume and critical charge definitions for Spin-Transfer Torque Magnetic Tunnel Junction. Thermal spike model is adopted to estimate the temperature reached during heavy ion irradiation and temperature effects are suggested to be responsible for the magnetic properties degradation and for upset processes.
\end{abstract}

\section{INTRODUCTION}

Radiation effects research on semiconductors has been pursued since the 1960s, becoming an extremely vivid area of research and development.

Breakthrough discoveries have occurred quite regularly, opening new perspectives for applications. Concepts like Linear Energy Transfer (LET), Critical Charge ( $\mathrm{Q}_{\mathrm{c}}$ ) and Sensitive Volume (SV) were defined to assess radiation effects on memory devices that store data by injecting or removing charge. Moreover, they were introduced at a time when the channel length of a transistor was in the order of few microns. While recognizing a long history of successful engineering practice based on these concepts, they now do show their limits for devices with nanometric dimensions and when the data storage mechanism is not based on charge.

This is the case of the Spin-Transfer Torque Magnetic Tunnel Junction (STT-MTJ), a magnetic storage device consisting of several layers of metals, ferromagnetic-alloys and insulator, with thicknesses varying from tens to fraction of nanometers. This peculiarity makes the scenario even more intricate. Indeed, the urgence of a careful evaluation of the materials surrounding the sensitive volume has been claimed in [1] [2] [3] since electronic and nuclear scattering in these regions can eventually contribute to Single Event Upset count (SEU). Additionally, the thermal effects induced by heavy ion energy deposition have been largely ignored in the field of radiation effects in electronics. Nevertheless, these effects will become more significant in electronic structures when the characteristic feature sizes will be reaching deeper into the nano-scale. Some studies tried to explain a posteriori the effects on MTJ irradiation sites by means of traditional LET cross-section plot or by exploiting TEM microscopy [4] [5].

In this paper, new insights are proposed to bring clear understanding on the possible basic degradation mechanisms that can trigger SEU in STT-MTJ, regardless of the radiation source, building a bridge between radiation effects and spintronic theory. By focusing on MTJ parameters such as specific heat, density and capacitance, we propose a sensitive volume definition for the MTJ that includes not only the $\mathrm{MgO}$ oxide barrier and both CoFeB layers, but the entire MTJ. Concerning the SEU triggering events in the CoFeB, two switching mechanisms are considered: Spin-Transfer Torque and thermal activation. For the first time, the temperature reached during the radiation-induced thermal spike is investigated. Indeed, the thermal stability of the material's intrinsic magnetization at temperatures below its Curie temperature is a key factor to determine its resistance to radiation. Additionally, since the dimensions of the MTJ are comparable to the distance between two MTJs in the same metal layer (as small as 500-750 $\mathrm{nm}$ in the most advanced nodes), recoil atoms from one magnetic device could upset its neighbor. This investigation on the fundamental mechanisms triggered in MTJ by irradiation are accompanied by a quantitative simulation analysis. Tradcare, a Geant 4 based simulations tool, was employed to this aim. A sketch of the Tradcare simulation flow is illustrated in Fig. 1.

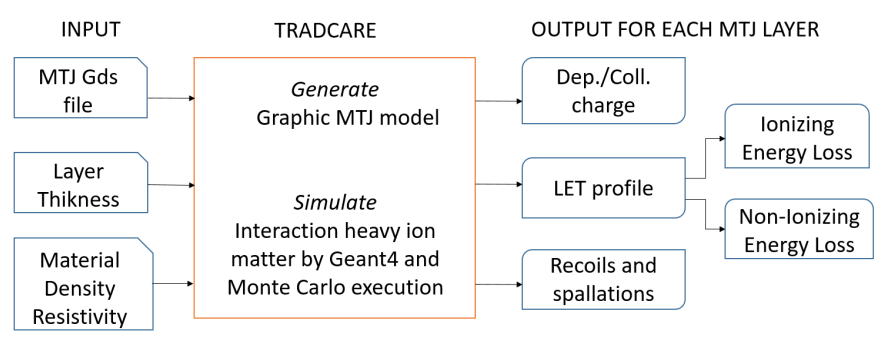

Fig. 1. Schematic view of the Tradcare flow

\section{Single Event EfFects originates in CoFeB}

\section{A. Spin-Transfer Torque Switching}

An STT-MRAM cell is composed by an MTJ consisting of a pinned $\mathrm{CoFeB}$ ferromagnetic reference layer (RL) and a free $\mathrm{CoFeB}$ ferromagnetic storage layer (FL) separated by a tunnel insulating barrier. Below the RL is placed a synthetic antiferromagnetic layer (SAF) composed of [Co $(0.50) / \mathrm{Pt}(0.25]_{3}$ antiferromagnetically coupled through a thin $\mathrm{Ru}$ spacer $(0.9)$ to an other multilayer $\left[\mathrm{Co}(0.50) / \mathrm{Pt}(0.25]_{6}\right.$, where numbers in 
parentheses stand for layer thickness, expressed in nanometers. Fig. 2 (b) details the entire device stack. The SAF structure is important both to minimize the dipolar field that acts from the $\mathrm{RL}$ on the FL, by aligning them in the parallel configuration, and to increase the perpendicular magnetic anisotropy (PMA). This latter is the crucial effect responsible for the correct spin switching dynamics and it originates at $\mathrm{CoFeB} / \mathrm{MgO}$ $\mathrm{MgO} / \mathrm{CoFeB}$ and $\mathrm{Co} / \mathrm{Pt}-\mathrm{Pt} / \mathrm{Co}$ interfaces, as Fig. 2 (c) clarifies. The final magnetic orientation has to be perpendicular to the plane in contrast to the parallel one, typical of the first STTMTJ generation [6].

The MTJ is designed in such a way that the magnetization of the storage layer $\vec{M}_{2}$ has two equilibrium positions: parallel or antiparallel to the RL magnetization $\vec{M}_{1}$. The transfer of spin is an interfacial effect, used to destabilize either one of these positions by modifying the local magnetization direction. Consequently, this transfer of spin tends to align the magnetization $\vec{M}_{2}$ along the direction of the spin current polarization, hence parallel (antiparallel) to $\vec{M}_{1}$ if the electron flow from RL (FL) to FL (RL).

The resistance of the MTJ cell depends on this relative magnetization alignment: as a result of the Tunnel Magnetoresistance (TMR) effect for ferromagnetic materials, $\mathrm{R}_{\mathrm{AP}}$ will always be higher that $R_{P}$ and this latter represents the most favoured configuration from the viewpoint of energetic consideration. The hysteresis loop of the FL is illustrated in Fig. 2 (a), where a field (coercive field, $\mathrm{H}_{\mathrm{c}}$ ) instead of a current is used, in equivalent way , to switch from bit " 1 " to bit " 0 ". It is worth to emphasize that the curve is symmetrical and the offset field is 0 thanks to the SAF compensation effect.
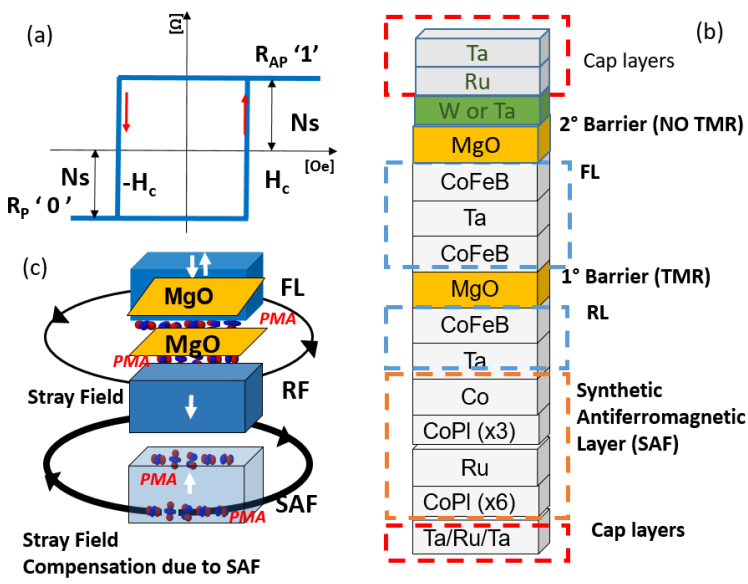

Fig. 2. Hysteresis loop of the Free Layer: $\mathrm{H}_{\mathrm{c}}$ represent the coercive field, $\mathrm{N}_{\mathrm{s}}$ the number of spin polarized electrons (a). Typical double-interface $\mathrm{CoFeB} / \mathrm{MgO}$ stack of a STT-MTJ (b). Simplified view of the three main blocks which constitute the STT-MTJ.

As demonstrated in [7] the minimum charge needed to switch the free ferromagnetic layer through an ordinary spin-torque mechanism is given by:

$$
Q_{\text {switch }}>2 q N_{\mathrm{s}}
$$

where $\mathrm{q}$ is the elementary electron charge and, $\mathrm{N}_{\mathrm{s}}$, representing the total number of spin, can be calculated as:

$$
N_{\mathrm{s}}=\frac{M_{\mathrm{s}} V}{\mu_{\mathrm{B}}}
$$

where $M_{s}$ is the saturated Magnetization, $V$ corresponds to the FL Volume and $\mu_{B}$ is the Bohr magneton. The quantity of charge obtained from Eq. 1 should be multiplied by 1.25 to take into account the fact that the polarization of the electrons tunneling through the $\mathrm{MgO}$ barrier is about $80 \%$ [8]. We identified with this value the critical charge of the FL, as defined traditionally, the minimum amount of charge needed for an SEU occurrence triggered by STT mechanism.

For example, for an MTJ with a radius of $10 \mathrm{~nm}$, we obtain $\mathrm{N}_{\mathrm{s}}=4062 \cdot 10^{3}$ and thus a value of $Q_{\text {criticFL }}=1,627 \mathrm{pC}$. According to Eq. 2, MTJ's diameter scaling and FL thickness decrease will both have a detrimental effect on the critical charge as shown in Fig. 3. Hence, we can compare $Q_{\text {switch }}$ (needed to trigger an upset by STT mechanisms) with the charge deposited in the FL by different ions at various energies to see if it can be exceeded. Simulation is used to evaluate the number of hits induced by $1 \mathrm{GeV} \mathrm{Xe}$ or $\mathrm{Ba}$ ions needed to deposit an amount of charge equal to $Q_{\text {criticFL }}$ as highlighted in Fig. 3. It is important to underline that, to trigger the requested current injection, these hits should occur quasi-simultaneously since the relaxation time of the excited carriers is in the order of ps [9]. It therefore appears very unlikely that direct hits induced by heavy ion could trigger an SEU. This is also highlighted by the STT-MTJ switching probability distribution obtained by electrical simulation and reported in Fig. 4: the duration of the switching pulse is around $2 n s$ when the applied voltage across the junction is $1 \mathrm{~V}$. It follows that, even if a shorter pulse could carry the same amount of charge, this would result in a voltage drop higher than $1 \mathrm{~V}$ across the MTJ, while the breakdown voltage of these devices is around $1.4 \mathrm{~V}$. In other words, a smaller number of more energetic strikes will cause directly the breakdown of the device instead of triggering a spin-transfer torque effect.

In conclusion, it seems almost impossible to explain the postirradiation STT-MTJ switching, observed in previous studies [10] [11], from the viewpoint of STT dynamics induced by particle hits whatever the ion species chosen for the irradiation. As a consequence, the critical charge is not a suitable definition neither for switching nor for breakdown since the pulse duration and voltage drop across the MTJ are decisive parameters to be taken into account.

\section{B. Thermal Switching}

The energy released within the MTJ by a particle strike could potentially thermally activate the switching of the magnetization. In order to estimate the heating process due to heavy ion irradiation, different models were proposed in the last decades [12]. Among them, the thermal spike model [13] is the only one that works for all kinds of materials including semiconductors and insulators [14] [15]. According to this model, heat waves propagate from the heavy ion hit 


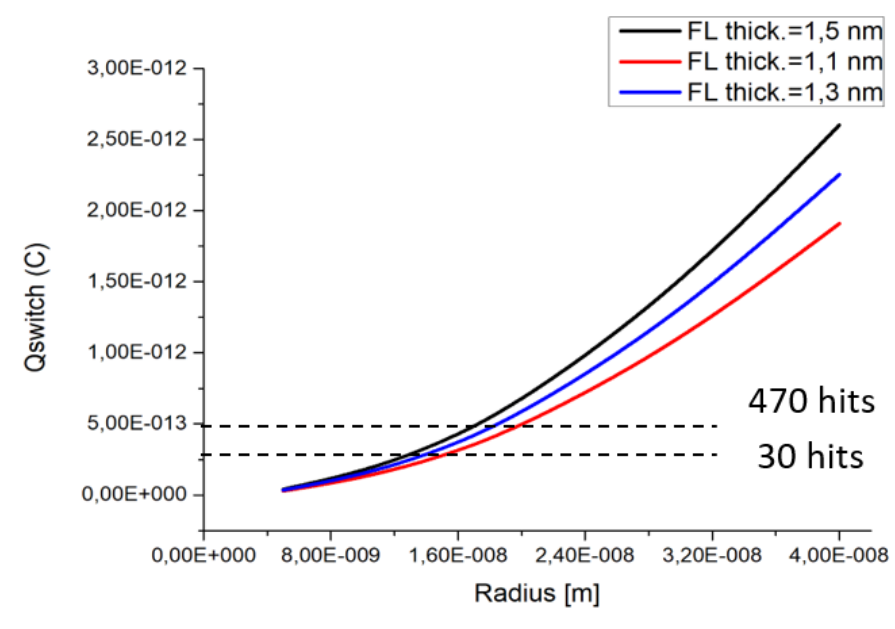

Fig. 3. Free layer Critical charge by varying MTJ radius and FL thickness

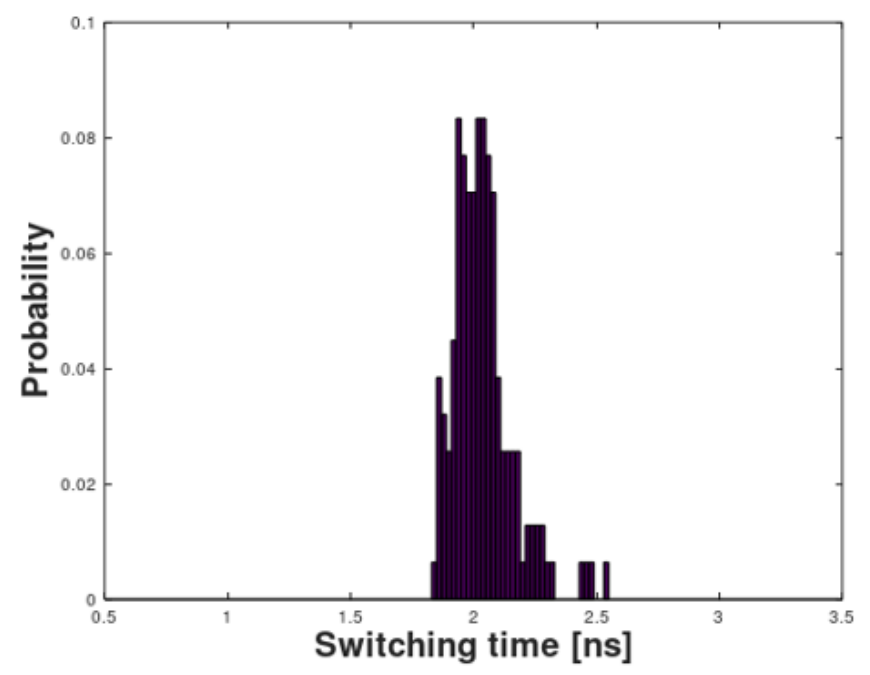

Fig. 4. Switching probability distribution at $1 \mathrm{~V}$ for $10 \mathrm{~nm}$ radius STT- MTJ

point around the ion track in a radial way. The main idea is to suppose that the energy deposited in the lattice can be described by a transient thermal process acting in the electronic and atomic subsystems. The temperature evolution in the spike can be calculated by assuming that the initial temperature distribution has the form of a $\delta$ function along a linear ion track [16]. Thence, the temperature at a radial distance $r$ from the impact and at time $t$ can be written in cylindrical coordinates as:

$$
T(\mathrm{r}, \mathrm{t})=\frac{F_{\mathrm{d}}}{4 \pi k t} \exp \left(-\frac{C r^{2}}{4 k t}\right)+T_{(\mathrm{t}=0)}
$$

where $F_{d}$ is the fraction of deposited energy in the material per unit length (electronic excitation and nuclear collisions), $K$ is the thermal conducivity, $C$ is the heat capacity and $T_{(t=0)}$ is the material's temperature before the stike. Density $(\rho)$ and heat capacity $(c)$ values for all the MTJ layers are listed in Table I. For metals as well as for alloys specific heat capacities were estimated based on the Dulong Petit law. $F_{d}[\mathrm{KeV} / \mathrm{nm}]$
TABLE I

VALUES OF DENSITY $\rho$, HEAT CAPACITY $c$, AND TOTAL THICKNESS $t$, OF THE MTJ LAYERS MATERIALS

\begin{tabular}{cccc}
\hline \hline Material & $\rho\left[\mathrm{Kgm}^{-3}\right]$ & $c[\mathrm{~J} /(\mathrm{k} \mathrm{Kg}]$ & $t[\mathrm{~nm}]$ \\
\hline $\mathrm{Ta}(\beta)$ & 16327 & 144 & 178.3 \\
$\mathrm{Pt}$ & 21450 & 180 & 4.25 \\
$\mathrm{Co}$ & 8900 & 639 & 5 \\
$\mathrm{Ru}$ & 12370 & 239 & 1.6 \\
$\mathrm{CoFe}$ & 8658 & 446 & 2.7 \\
$\mathrm{MgO}$ & 3560 & 648 & 1.2 \\
\hline \hline
\end{tabular}

was computed from the LET profile obtained from Tradcare simulations. Fig. 5 depicts the LET at $1 \mathrm{GeV}$ for $\mathrm{Xe}$ and $\mathrm{Ba}$ ions across the three MTJ's layers: FL, insulator, RL. It is

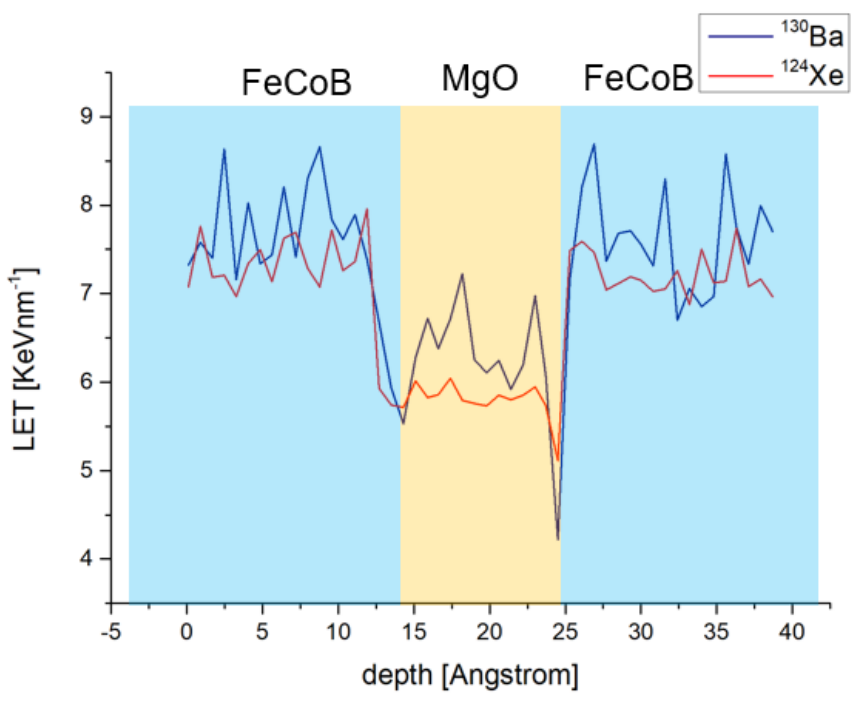

Fig. 5. Electronic LET profile for Ba and Xe ions across the three MTJ's layes FL ( $\mathrm{FeCoB})-\mathrm{MgO}-\mathrm{RL}(\mathrm{feCoB})$.

obvious that the electronic stopping power affects the location of the range distribution. Therefore, the thermal spike's strength depends on the ion type and on the material where it originates. Fig. 6 shows the temperature distribution for Xenon ion on $\mathrm{FeCoB}$. Extremely high temperature (thousands of kelvin) are reached during some fs to few ps at the strike point. Then, the heat waves continue to propagate: as shown in the inset of Fig. 6 from 1 ns to 2 ns a temperature of $370 \mathrm{~K}$ is reached regardless of the distance $(3 \mathrm{~nm}$ to $10 \mathrm{~nm}$ ) from the hit point. After 2 ns to 4 ns the temperature decrease rate is less pronounced and an homogeneous value of $340 \mathrm{~K}$ stabilizes. Simulation demonstrate that particle strikes on $\mathrm{Si}$ substrate can thermally affect the MTJ. In addition, the thermal spike propagates, thus the MTJ does not even need to be directly hit to be reached by an undesired thermal spike. Indeed, if we called maximum propagation range the radial distance at which the temperature returns to around $300 \mathrm{~K}$, we can observe in Fig. 7 that this value is way higher than a few $\mathrm{nm}$. A strike originating in Silicon has a maximum propagation range of $150 \mathrm{~nm}$ while the value of $200 \mathrm{~nm}$ is easily reached, for 


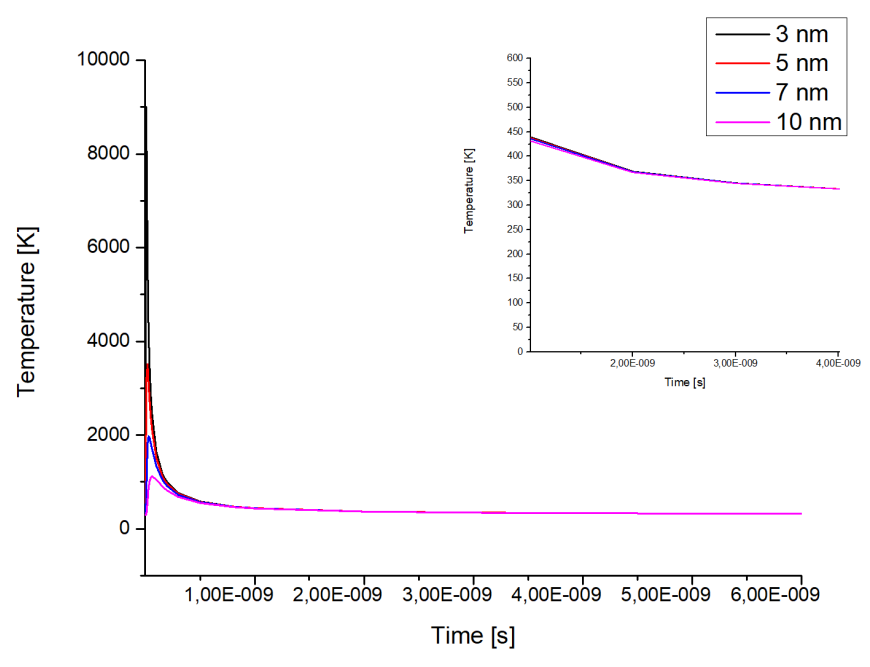

Fig. 6. Thermal spikes in FeCoB FL induced by a Xe strike, Inset: Temperature evolution in the window 1-4 ns.

example, in the case of a strike on a Platinum layer. However,

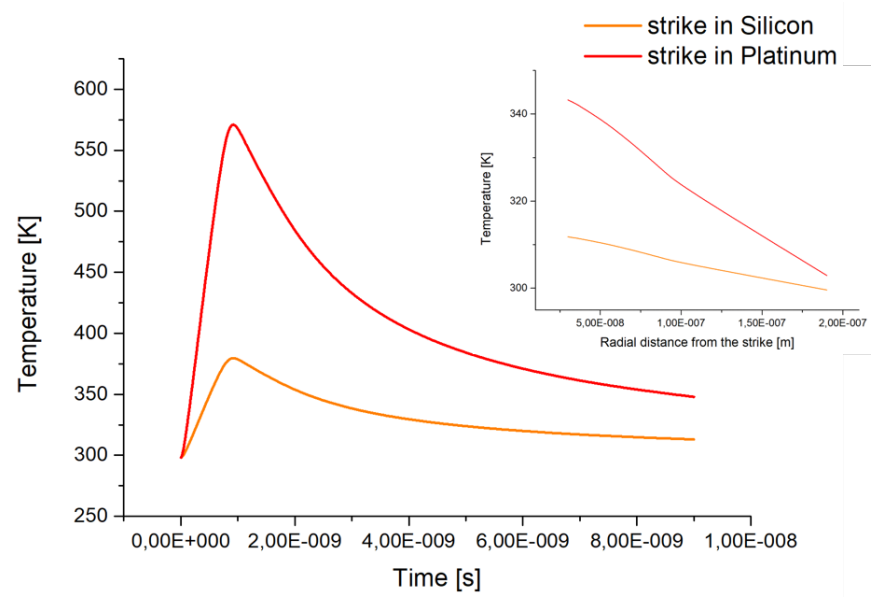

Fig. 7. Xe on $\mathrm{Si}$ and Pt: Thermal spikes at $30 \mathrm{~nm}$ from the hit point, Inset: maximum propagation range in $\mathrm{Si}$ and $\mathrm{Pl}$.

at a temperature $T$, the thermal switching probability of the magnetization, after a time $t$, is fitted exactly by the Néel Brown relaxation formula:

$$
P(t)=1-\exp \left(-\frac{t}{\tau_{0} \exp \left(-\frac{\Delta E}{K_{\mathrm{B}} T}\right)}\right)
$$

where the exponential at the denominator is the mean time needed to switch the MTJ's FL orientation, according to Arrhenius law: $\tau_{0}$ is the attempt period ( $\left.1 \mathrm{~ns}\right)$, and $\mathrm{T}$ is the temperature reached during the strike. As plotted in Fig. 8 for one bit, in a range of temperature between $400 \mathrm{~K}$ and $550 \mathrm{~K}$, the switching probabilities are less than $10^{-2}$. At higher temperatures they exponentially rise from $T=619 \mathrm{~K}$ and a corresponding $P_{\text {switch }}=8 \%$ to $T=750 \mathrm{~K}$ with $P_{\text {switch }}=50 \%$.

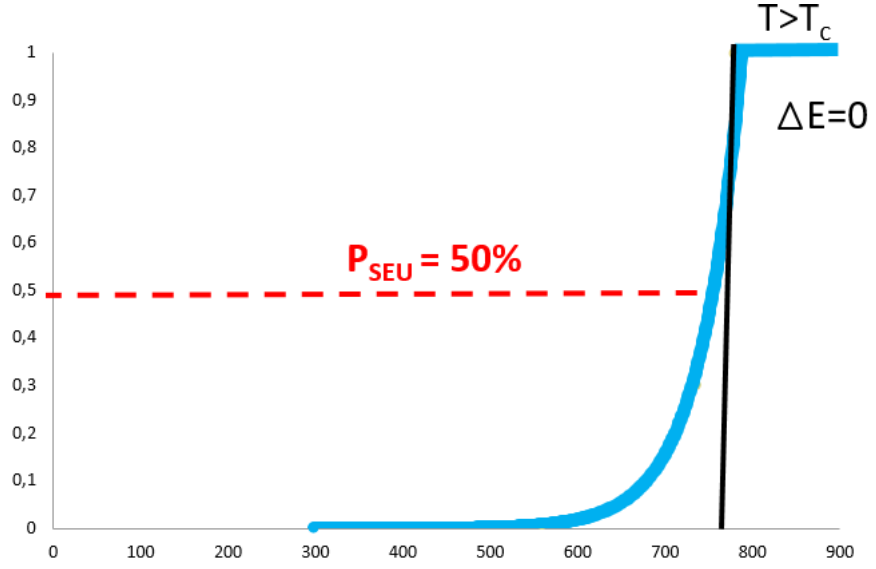

Fig. 8. Free layer thermal switching probability distribution after a time $\mathrm{t}=$ $5 \mathrm{~ns}$ at different temperatures

\section{TRANSIENT THERMAL EVENTS}

\section{A. Magnetic properties degradation}

Regardless of how the MTJs experience the temperature rise (direct hit or hit proximity), the magnetic properties of the spintronic devices are dramatically affected by these temperature spikes. These degradation effects are transient but their ability to threaten the memory should not be underestimated since they start to be triggered at temperature spikes not very high above room temperature. For this reason, it reasonable to expect that thermal induced stress, due to several heating/cooling cycles, could have an important impact on the functional STT-MTJ lifetime.

Indeed, according to Bloch's low [17], the temperature dependency of the saturation magnetization $M_{S}$ and the anisotropy $K_{u}$ can be fitted as:

$$
\begin{aligned}
& M_{\mathrm{S}}(t)=M_{\mathrm{s}}(0)\left[1-\left(\frac{T}{T \mathrm{c}}\right)^{3 / 2}\right] \\
& K_{\mathrm{u}}(t)=K_{\mathrm{u}}(0)\left[\left(\frac{M_{\mathrm{s}}(t)}{M_{\mathrm{s}}(0)}\right)^{2.2}\right]
\end{aligned}
$$

As noticeable in Fig. 9 b, perpendicular anisotropy is more sensitive to temperature rise with respect to magnetization (Fig. 9 a). However, among the STT-MTJ analysed parameters, the coercive field is the most affected by temperature increase, as our experimental data in Fig. $9 \mathrm{c}$ demonstrate, in agreement with the state of the art [18]. For example, a temperature of 340 $\mathrm{K}$ is already high enough to decrease the anisotropy by $17 \%$ whereas at the same temperature the coercive field is reduced by $38 \%$ from its initial value. Around $510 \mathrm{~K}$ the transient loss of $M_{s}$ and $K_{v}$ is attested to be $50 \%$ of their initial value.

\section{B. Thermal stability and Retention time of the stored infor- mation}

The energy barrier, $\Delta \mathrm{E}$, which divides the two stable opposite states (AP, P), depends in a complex way on both $\mathrm{M}_{\mathrm{s}}$ and $\mathrm{K}_{\mathrm{u}}$ (and on the free layer volume) [19]. Nonetheless, 

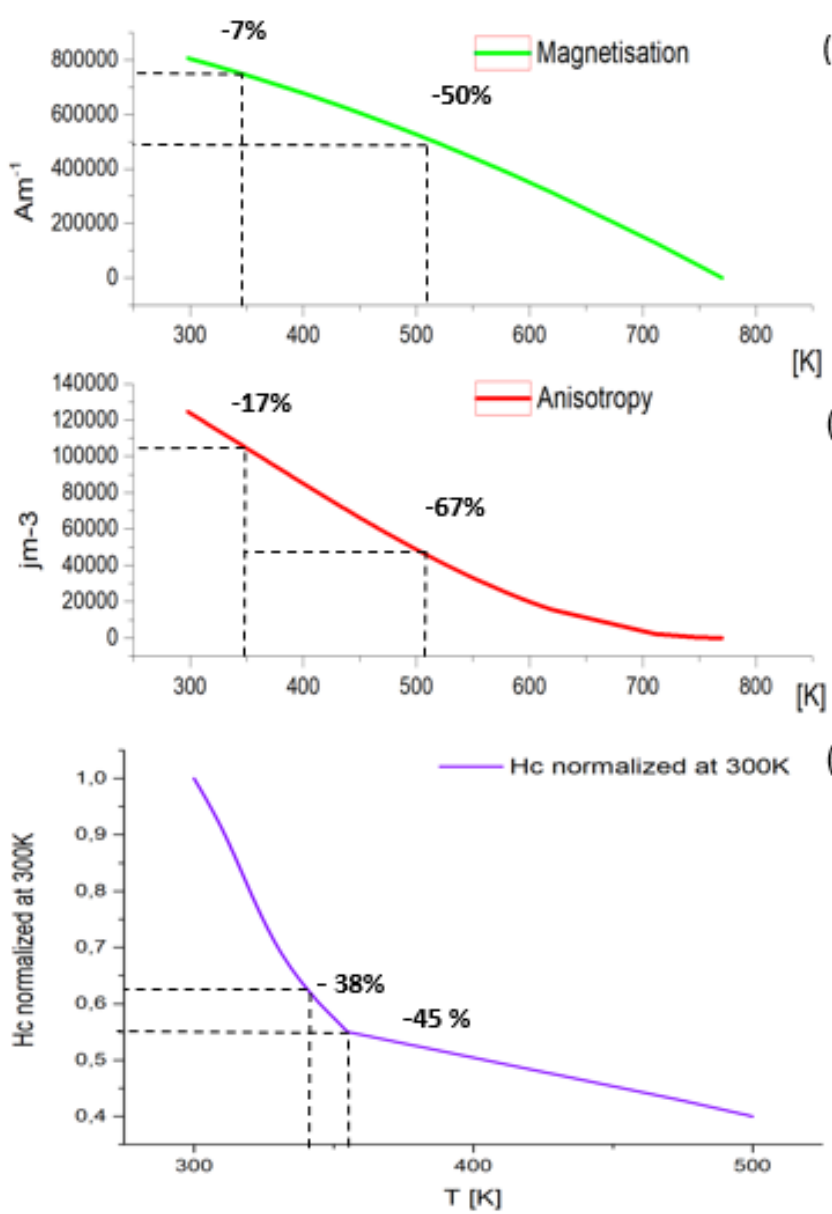

Fig. 9. Temperature variation of Magnetization (a), Anisotropy (b) and Coercive field (c)

in macrospin regime $(\mathrm{r}<30 \mathrm{~nm})$ the barrier height, could be approximated, in a simplified way, as:

$$
\Delta E=K_{\mathrm{u}} V
$$

where $K_{v}$ is the anisotropy and $V$ is the FL volume. Then, recalling that $K_{B}$ is the Boltzman constant, we can define an important parameter which represents the MTJ's thermal stability factor, as follows:

$$
\Delta=\frac{\Delta E}{K_{B} T}
$$

It follows immediately that a temperature rise, combined with the radius scaling, will drastically reduce the thermal stability of the memory device, as the plots in Fig. 10 illustrate. The stability of the stored information (i.e. the retention time) for a duration $T_{r}$ can be written as [20]:

$$
\frac{\Delta E}{K_{\mathrm{B}} T}>\ln \left(\frac{T_{\mathrm{r}}}{\tau_{0}}\right)
$$

This inequality sets the condition upon the minimum barrier height needed for the stability of the storage layer against thermal fluctuations at a given $T$. For example, for a 3 years space mission, we obtain from Eq. 9: $\Delta \mathrm{E}>39 K_{B} T$ that is not

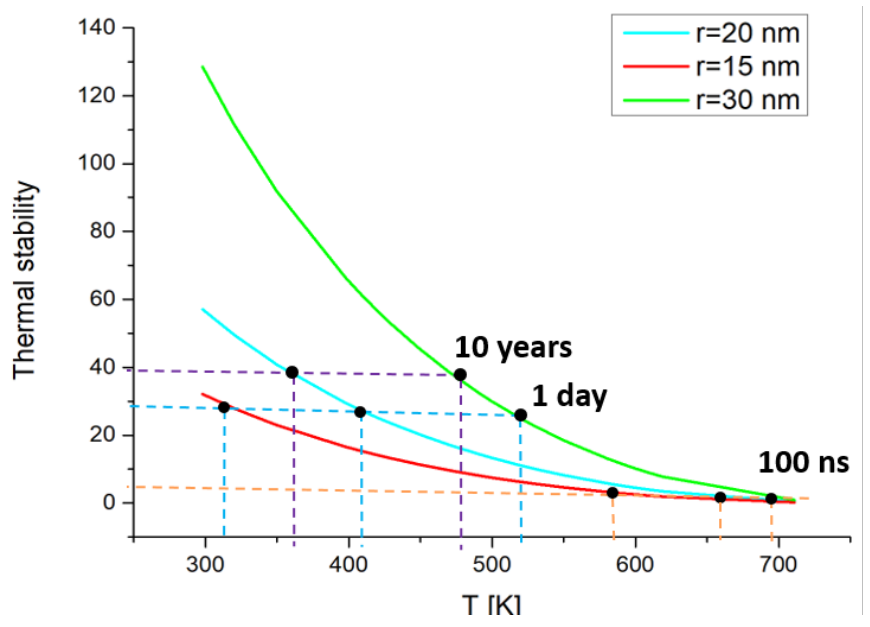

Fig. 10. Thermal stability factor and retention time impacted from scaling and temperature rise

met for any temperature above $353 \mathrm{~K}$. Luckily enough, the temperature of the MTJ will not be constantly equal to the one reached during the strike, but a criticity still remains since MTJ scaling also has a deleterious impact on the thermal stability factor as highlighted in Fig. 10. This could represent an important limitation for missions where the average temperature at device level is expected to be constantly higher than $350 \mathrm{~K}$. Indeed, this failure mechanism is related to temperature, hence could be triggered even without any particle strikes. Additionally, these considerations suggest that the most scaled devices are not the most suitable for harsh environment application. An interesting idea to overcome this problem could be the recently presented Perpendicular Shape Anisotropy (PSA) MRAM in which the dimension (volume) of the free layer is drastically increased along the vertical axis while keeping a scaled radius [21]. Another promising option to enlarge $\Delta E$ is to increase the number of $\mathrm{MgO}$-ferromagnetic interfaces [22].

\section{Permanent thermal efFects}

\section{A. $H_{C}$ degradation and the role of Synthetic Antiferromagnetic layer}

The narrowing of the hysteresis loop in perpendicular anisotropy STT-MTJ as temperature rises was already experimentally proven in [23]. In this work, a mismatch was found among the theoretical prediction of the $H_{C}$ temperature degradation rate in [24] and the obtained experimental data. For this reason, it was concluded that the temperature sensitivity of $H_{C}$ was in reality higher than the one predicted in [24] due to stress or strain on the devices, caused by the patterning and encapsulation. Here we want to take one step beyond, and argue that, even after the cool down, some degradation in the coercive field remain.

This was observed by measuring the hysteresis loop of some STT-MTJ SPINTEC samples before and after a bake at $200^{\circ} \mathrm{C}$ : the coercive field is reduced as plotted in Fig. 11 a. Since 
the absolute value of the coercive field appears to be reduced mainly from AP to $\mathrm{P}$ state transition, the thermal rise seemed to induce an asymmetry of thermal stability between parallel and antiparallel states, the first one being favored. Interestingly, the simultaneous variation observed in offset field, $H_{\text {off }}$ (Fig. 11 b) allows us to argue that the first is a consequence of the second: defects creation in the SAF results in an uncompensated stray magnetic field from RL to FL that ends up favoring the transition towards parallel state, i.e., a smaller absolute value of the coercive field from AP to P reversal. These findings are in agreement with [25] where it was proven, experimentally and for the first time, not only that $H_{\text {off }}$ and $H_{c}$ changes are related, but also that a significant increase of the first induced a reduction of the second and this correlation worsen with scaling.

In conclusion, even if the temperature rise associated with a single event lasts for a short time, the effects on the MTJ stability could be permanent, most likely cumulative, due to a complicated sinergy of recoils and electronic excitation such as cascade collisions, thermal spikes, latent track formation and displacement damage. As a result, an important role is played by the different coefficient of thermal expansion inside the MTJ (magnetovolume) and by the materials that surround the MTJ and could exert a strain on it (magnetostriction). The relation between thermal film stress/strain and magnetic properties in $\mathrm{FeCoB}$ is particularly strong since these alloys have a high positive magnetostriction [26]. Additionally, experiments found out that magnetovolume and magnetostriction result also in a modification of Curie Temperature for the ferromagnetic materials: an increase in case of lattice expansion and a decrease in case of striction. In the case of swift heavy ions irradiation, the thermal MTJ's bottleneck could be most likely represented by the SAF, the two Co/Pt multi-layers blocks separated by the Ru spacer. There are at least two motivations to this statement. The first one is related to its thickness and density: according to our simulation, most of the energy will be dissipated in this part of the MTJ. Our findings are in accordance with [27] where after swift heavy ion irradiation, the STT-MTJ most affected layer was the Co/Pt. Noticeably, as shown previously (see Fig. 7), the radial distribution of the heat propagation for the Pt is very wide. The second is related to the key role of the Rutenium spacer that has to ensure the antiferromagnetic coupling between the two CoPl multilayers: a reduction in the effective thickness of the $\mathrm{Ru}$ $(0.9 \mathrm{~nm})$ (due for example to thermal intermixing or diffusion of $\mathrm{Ta}$ [28], or Fe in the SAF [29] ) will result in a loss of the SAF magnetic moment and thus a destabilization of the AP state. This possibility is corroborated by [28] where it is demonstrated that over a fluence of $10^{14}$ ions $/ \mathrm{cm}^{2}$ the 2 blocks become ferromagnetically coupled and behave as a single thicker ferromagnetic layer. Accordingly, the loss of perpendicular Magnetic Anisotropy was documented in CoPt multilayers over $10^{15} \mathrm{Ga}^{+}$ions $/ \mathrm{cm}^{2}$ irradiation in [30] and in [31]. In particular, the substitution of Ta, as the cap layer, with $\mathrm{W}$ seems to be beneficial to avoid deleterious material inter diffusion [32].

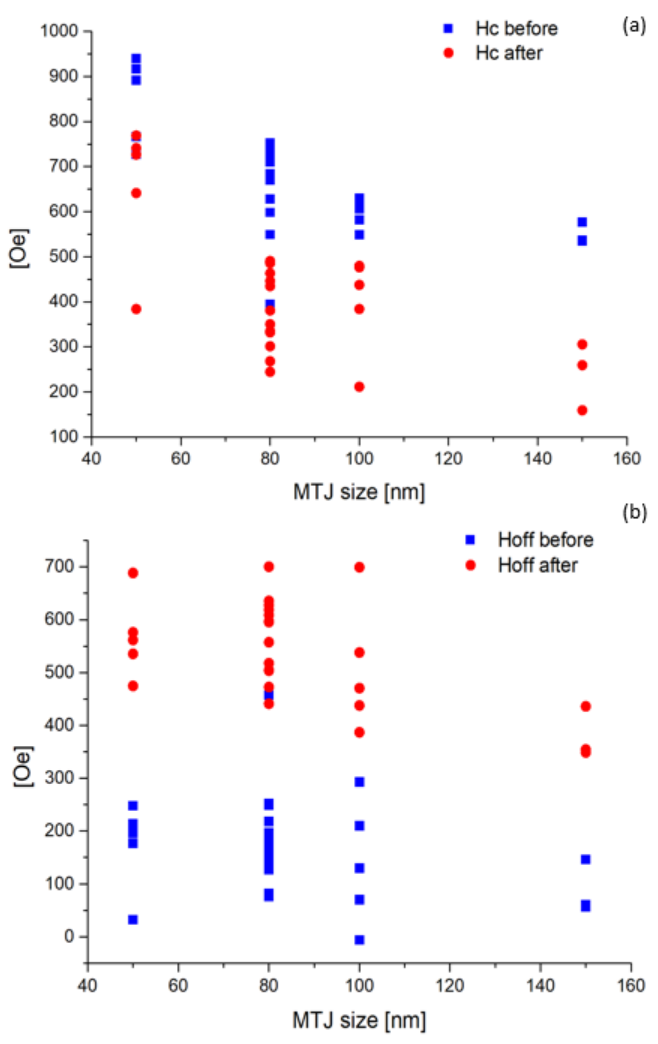

Fig. 11. Variation of coercive field and offset field post $200^{\circ} \mathrm{C}$ annealing

\section{B. Curie Temperature overtaking: Thermal Event Upset}

Thermal switching and temperature effects appearing to be a serious concern, one more important temperature-dependent processes should be analysed, since it could lead to the loss of the stored information.

Indeed, ferromagnetic materials preserve their magnetic property only below the so-called Curie temperature, above which they fall in a disordered magnetization state, becoming paramagnetic. If this threshold is exceeded even for a very short duration, the magnetization is completely destroyed and then rebuilt during cooling time, in principle, in up or down state with equal probability. In reality, since the parallel state is favored from an energetic point of view, the final mutual configuration of the FL and RL will always be parallel, regardless of which ferromagnetic layer's magnetization will be rebuilt first. In other words, this is an asymmetrical upset as the final state will always be logic value "0'. Thence, if the initial stored bit was " 1 ' the probability of upset is $100 \%$; on the contrary, if the initial stored bit is " 0 'the bit will be restored after a transient loss due to the paramagnetic transition. However, the MTJ will be still operational after cooling, but the information in the bit cell could be corrupted. The Curie temperature, specific for each material, strongly depends on the thickness of the considered layer: for a $1.5 \mathrm{~nm}$ thick CoFeB layer it is $770 \mathrm{~K}$ [33]. This could explain the sudden SEU occurrence always toward parallel state. This upset is not directly related to the strike of the MTJ but 
rather to the temperature reached even in the proximity of the device, exceeding the material's Curie's temperature. We propose to call this specific mechanism Thermal Event Upset (TEU).

The notion of proximity becomes particularly delicate since the device size is comparable to the distance between them. This latter is attested to be as small as $500 \mathrm{~nm}$ in the most advanced technology node. Fig. 12 shows the secondary energetic recoil products range and energies. Even in the presence of the typical $\mathrm{SiO}_{2}$ spacers that surround each MTJ, simulation results seem to suggest that the most energetic recoils could threaten the neighbour MTJ on the same metal layer.

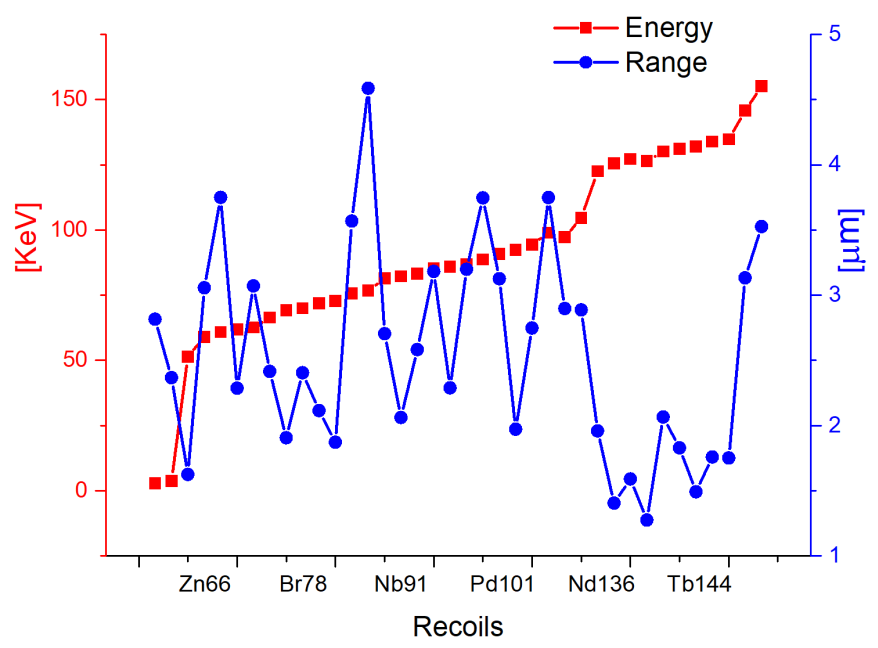

Fig. 12. Energy and longitudinal range of secondary particles produced by Xe ion strikes in the MTJ.

\section{MgO Tunnel Barrier}

If the thermally driven processes triggered under irradiation have a predominant role during ion strikes (in the limit of a certain fluence), it seems reasonable to argue that degradation of magnetic properties appear before degradation of electrical properties. Reference [34] presents irradiation results obtained in recent perpendicular STT-MTJ which corroborate our thesis. The observation has been first attributed to the modest penetration of the low energy ions in the device stack but, it has also been observed after high energy ( $\geq 2 \mathrm{GeV})$ ion irradiation [27]. For this last ion energy, the nuclear energy loss $(\leq 1 \mathrm{keV} / \mathrm{nm})$ is negligible compared to the electronic energy loss thence the inelastic collision dominates the energy loss process. Along the incident ion path, there is a state of intense electric excitation that, in principle, can lead to the formation of a damaged region and the electron-phonon coupling constant dominate how the thermal energy diffuse over time according to the thermal spike model (as detailed in Section II). However, highly ionizing particle irradiation in insulator can, via a thermal spike phenomenon, promote self-healing rather than defect production [35]. This could explain the observed robustness of the electric properties with respect to the magnetic ones, even when ion irradiation can induce the presence of few tracks observable in cross-sectional TEM image in the insulating $\mathrm{MgO}$ layer [27]. If the thermal spike is the dominant damaging process the same hypotheses formulated for the degradation of the magnetic properties explain the maintain of the electric ones. This should be valid at low and moderate fluences when the possibility to precisely hit the MTJ is intrinsically very low. This will also explain why with increasing fluence the TMR is finally corrupted: typically, numerous tracks creation occur in the $\mathrm{MgO}$ creating defect-rich regions. At high fluence, this degradation process dominates over self-healing. The ion type chosen for the irradiation still plays a key role since the difference in mass and thus nuclear and electric stopping power in the material lead to important fluctuations, as confirmed by simulation results summarized in Table II.

\section{CONCLUSION}

In this paper, we propose new interpretations for some traditional radiation-induced parameters, trying to translate them in the Beyond-CMOS paradigm of spintronics.

We show that explaining the STT-MTJ bit flip associated to particle hits with Spin-Transfer Torque switching mechanism in $\mathrm{CoFeB}$ is almost impossible. Thermal effects were suggested instead to be responsible of bit flip and MTJ magnetic properties degradation. Thermal spike model was used to estimate the temperature reached during irradiation and its radial extension. This was possible using data such as MTJ LET profile, deposited charge, and secondary ion production, data provided by STT-MTJ model simulation in Tradcare environment.

Thermal event upset (TEU) is a proposed new nomenclature to describe an asymmetrical upset, always resulting in logic state '0", that occurs each time the MTJ temperature exceeds the Curie temperature. Thermal stability factor is the other critical parameter, since strictly related to the required retention time and dramatically affected by MTJ size scaling and temperature rise. Moreover, thermally-induced switching probability is estimated a serious concern since thermal spike models suggests heat waves can propagate for hundreds of nanometers. Therefore after an ion hit, the MTJ temperature could be increased without being directly hit by the incident ion. Even before the occurrence of a TEU, our results show that the main magnetic MTJs properties can be affected even at temperature. below $T_{c}$. These considerations lead to extend the sensitive volume definition over the entire MTJ stack. During irradiation, magnetic properties degradation occurs before electrical property degradation in agreement with the thermal driven process: the temperature effect that could induce a selfhealing in the $\mathrm{MgO}$, preserving its crystallinity and thus the MTJ's electrical properties is, on the other hand, deleterious for the magnetic properties. In conclusion, this study highlights the need of new STT-MTJ stack solution, specific for harsh environment, to mitigate the deleterious impact of scaling on STT-MTJ thermal stability. Additionally, as the FL CoFeB 
TABLE II

NON IONIZING ENERGY LOSS (NIEL), DISPLACEMENT PER ATOMS AND NUMBER OF ATOMS DISPLACED IN THE MGO TUNNEL BARRIER UNDER 1 GEV IRRADITION, $10^{12}$ IONS/CM ${ }^{2}$

\begin{tabular}{cccc}
\hline \hline & NIEL $[\mathrm{KeV} / \mathrm{nm}]$ & DPA $\left[10^{-4}\right]$ & Dispaced Atoms \\
\hline $\mathrm{Xe}$ & 3.58 & 3 & 56 \\
$\mathrm{Ba}$ & 4.29 & 3.6 & 68 \\
$\mathrm{Bi}$ & 12.8 & 11.7 & 221 \\
\hline \hline
\end{tabular}

thickness shrinks even more its Curie temperature decreases: inducing a TEU thus becomes even easier and more probable. Therefore, with device scaling multiple TEUs could occur as technology gets denser and neighbour MTJ closer to one another.

\section{REFERENCES}

[1] P. E. Dodd, J. R. Schwank, M. R. Shaneyfelt, J. A. Felix, P. Paillet, V. Ferlet-Cavrois, J. Baggio, R. A. Reed, K. M. Warren, R. A. Weller, R. D. Schrimpf, G. L. Hash, S. M. Dalton, K. Hirose, and H. Saito, "Impact of heavy ion energy and nuclear interactions on single-event upset and latchup in integrated circuits," IEEE Transactions on Nuclear Science, vol. 54, no. 6, pp. 2303-2311, 2007.

[2] R. A. Reed, R. A. Weller, R. D. Schrimpf, M. H. Mendenhall, K. M. Warren, and L. W. Massengill, "Implications of nuclear reactions for single event effects test methods and analysis," IEEE Transactions on Nuclear Science, vol. 53, no. 6, pp. 3356-3362, 2006.

[3] K. M. Warren, R. A. Weller, M. H. Mendenhall, R. A. Reed, D. R. Ball, C. L. Howe, B. D. Olson, M. L. Alles, L. W. Massengill, R. D. Schrimpf, N. F. Haddad, S. E. Doyle, D. McMorrow, J. S. Melinger, and W. T. Lotshaw, "The contribution of nuclear reactions to heavy ion single event upset cross-section measurements in a high-density seu hardened sram," IEEE Transactions on Nuclear Science, vol. 52, no. 6, pp. 2125-2131, 2005.

[4] D. Kobayashi, Y. Kakehashi, K. Hirose, S. Onoda, T. Makino, T. Ohshima, S. Ikeda, M. Yamanouchi, H. Sato, E. C. Enobio, T. Endoh, and $\mathrm{H}$. Ohno, "Influence of heavy ion irradiation on perpendicularanisotropy cofeb-mgo magnetic tunnel junctions," in 2013 14th European Conference on Radiation and Its Effects on Components and Systems (RADECS), 2013, pp. 1-5.

[5] J. Lu, S. J. Poon, S. A. Wolf, B. D. Weaver, P. J. McMarr, H. Hughes, and E. Chen, "Radiation effects on the magnetism and the spin dependent transport in magnetic materials and nanostructures for spintronic applications," Journal of Materials Research, vol. 30, no. 9, p. 1430-1439, 2015.

[6] S. Ikeda, K. Miura, H. Yamamoto, K. Mizunuma, H. Gan, M. Endo, S. Kanai, J. Hayakawa, F. Matsukura, and H. Ohno, "A perpendicularanisotropy cofeb-mgo magnetic tunnel junction," Nature materials, vol. 9, pp. 721-4, 092010 .

[7] B. Behin-Aein, A. Sarkar, S. Srinivasan, and S. Datta, "Switching energy-delay of all-spin logic devices," Applied Physics Letters, vol. 98, p. 123510, 032011.

[8] R. Matsumoto, A. Fukushima, T. Nagahama, E. Tamura, Y. Suzuki, K. Ando, and S. Yuasa, "Quantitative analysis of coherent and incoherent tunneling currents in MgO-based epitaxial magnetic tunnel junctions," Japanese Journal of Applied Physics, vol. 50, no. 6, p. 063003, jun 2011. [Online]. Available: https://doi.org/10.1143\%2Fjjap.50.063003

[9] D. Fleetwood, S. Pantelides, and R. Schrimpf, Defects in microelectronic materials and devices, 012008.

[10] D. Kobayashi, K. Hirose, T. Makino, S. Onoda, T. Ohshima, S. Ikeda, H. Sato, E. C. Enobio, T. Endoh, and H. Ohno, "Soft errors in 10nm-scale magnetic tunnel junctions exposed to high-energy heavy-ion radiation," Japanese Journal of Applied Physics, vol. 56, p. 802, 2017.

[11] R. R. Katti, "Radiation-induced errors at elevated linear energy transfer levels and magnetic error rate interactions in magnetic tunnel junctions," in 2019 IEEE Radiation Effects Data Workshop, 2019, pp. 1-4.
[12] M. Toulemonde, C. Dufour, Z. Wang, and E. Paumier, "Atomic and cluster ion bombardment in the electronic stopping power regime: A thermal spike description," Nuclear Instruments and Methods in Physics Research Section B: Beam Interactions with Materials and Atoms, vol. 112, no. 1, pp. 26 - 29, 1996, new Trends in Ion Beam Processing From Ions and Cluster Ion Beams to Engineering Issues. [Online]. Available: http://www.sciencedirect.com/science/article/pii/0168583X95011293

[13] G. Szenes, "Ion-velocity-dependent track formation in yttrium iron garnet: A thermal-spike analysis," Phys. Rev. B, vol. 52, pp. 61546157, Sep 1995. [Online]. Available: https://link.aps.org/doi/10.1103/ PhysRevB.52.6154

[14] S. Daraszewicz and D. Duffy, "Extending the inelastic thermal spike model for semiconductors and insulators," Nuclear Instruments and Methods in Physics Research Section B: Beam Interactions with Materials and Atoms, vol. 269, no. 14, pp. 1646 - 1649, 2011, computer Simulations of Radiation Effects in Solids. [Online]. Available: http://www.sciencedirect.com/science/article/pii/S0168583X10008566

[15] S. L. Daraszewicz and D. M. Duffy, "Extending the inelastic thermal spike model for semiconductors and insulators," Nuclear Instruments and Methods in Physics Research B, vol. 269, no. 14, pp. 1646-1649, Jul. 2011.

[16] M. Brongersma, E. Snoeks, and A. Polman, "Temperature dependence of mev heavy ion irradiation-induced viscous flow in sio2," Applied Physics Letters, vol. 71, pp. 1628-1630, 091997.

[17] N. Ashcroft and N. Mermin, Solid State Physics. Fort Worth: Saunders College Publishing, 1976.

[18] J. Iwata-Harms, G. Jan, H. Liu, S. Serrano-Guisan, J. Zhu, L. Thomas, R.-Y. Tong, V. Sundar, and P. Wang, "High-temperature thermal stability driven by magnetization dilution in cofeb free layers for spin-transfertorque magnetic random access memory," Scientific Reports, vol. 8, 2018.

[19] S. Lequeux, N. Perrissin, G. Gregoire, L. Tillie, A. Chavent, N. Strelkov, L. Vila, I. Prejbeanu, S. Auffret, R. Sousa, L. Buda-Prejbeanu, D. R. Enrico, E. Gautier, A. Conlan, D. Cooper, and B. Dieny, "Thermal robustness of magnetic tunnel junctions with perpendicular shape anisotropy," Nanoscale, vol. 12, 022020.

[20] B. Dieny, R. Goldfarb, and K.-J. Lee, Introduction to magnetic randomaccess memory, 112016.

[21] N. Perrissin, S. Lequeux, N. Strelkov, A. Chavent, L. Vila, L. D. Buda-Prejbeanu, S. Auffret, R. C. Sousa, I. L. Prejbeanu, and B. Dieny, "A highly thermally stable sub-20 nm magnetic random-access memory based on perpendicular shape anisotropy," Nanoscale, vol. 10, no. 25, p. 12187, 2018. [Online]. Available: https://hal.archives-ouvertes.fr/hal-01824092

[22] K. Nishioka, H. Honjo, S. Ikeda, T. Watanabe, S. Miura, H. Inoue, T. Tanigawa, Y. Noguchi, M. Yasuhira, H. Sato, and T. Endoh, "Novel quad interface mtj technology and its first demonstration with high thermal stability and switching efficiency for stt-mram beyond 2xnm," in 2019 Symposium on VLSI Technology, 2019, pp. T120-T121.

[23] C. Park, J. J. Kan, C. Ching, J. Ahn, L. Xue, R. Wang, A. Kontos, S. Liang, M. Bangar, H. Chen, S. Hassan, S. Kim, M. Pakala, and S. H. Kang, "Temperature dependence of critical device parameters in $1 \mathrm{gb}$ perpendicular magnetic tunnel junction arrays for stt-mram," IEEE Transactions on Magnetics, vol. 53, no. 2, pp. 1-4, 2017.

[24] D. Suess, L. Breth, J. Lee, M. Fuger, C. Vogler, F. Bruckner, B. Bergmair, T. Huber, J. Fidler, and T. Schrefl, "Calculation of coercivity of magnetic nanostructures at finite temperatures," Physical Review B, vol. 84, 102011.

[25] H. Jiancheng, S. Hin, V. B. Naik, S. T. Lim, and G. Han, "Effect of the stray field profile on the switching characteristics of the free layer in a perpendicular magnetic tunnel junction," Journal of Applied Physics, vol. 117, p. 17B721, 052015.

[26] N. Minor and T. Klemmer, "Magnetic and structural properties of fecob thin films," Magnetics, IEEE Transactions on, vol. 37, pp. 2302 - 2304, 082001.

[27] B. Wang, Z. Wang, A. Du, Y. Qiang, K. Cao, Y. Zhao, H. Zheng, S. Yan, P. Zhai, J. Liu, G. Guo, Y. Bai, J. Wang, and W. ZHAO, "Radiation impact of swift heavy ion beams on double-interface cofeb/mgo magnetic tunnel junctions," Applied Physics Letters, vol. 116, p. 172401, 042020.

[28] B. Teixeira, A. Timopheev, N. Caçoilo, L. Cuchet, J. Mondaud, J. Childress, S. Magalhães, E. Alves, and N. Sobolev, "Ar + ion irradiation of magnetic tunnel junction multilayers: impact on the magnetic and electrical properties," Journal of Physics D: Applied Physics, 072020. 
[29] H. Honjo, S. Ikeda, H. Sato, T. Watanebe, S. Miura, T. Nasuno, Y. Noguchi, M. Yasuhira, T. Tanigawa, H. Koike, M. Muraguchi, M. Niwa, K. Ito, H. Ohno, and T. Endoh, "Origin of variation of shift field via annealing at $400^{\circ} \mathrm{C}$ in a perpendicular-anisotropy magnetic tunnel junction with $[\mathrm{Co} / \mathrm{Pt}]-$ multilayers based synthetic ferrimagnetic reference layer," AIP Advances, vol. 7, no. 5, p. 055913, May 2017.

[30] I. Sveklo, P. Mazalski, J. Jaworowicz, J.-P. Jamet, N. Vernier, A. Mougin, J. Ferré, M. Kisielewski, V. Zablotskii, E. Bourhis, J. Gierak, K. Postava, J. Fassbender, J. Kanak, and A. Maziewski, "Modification of Magnetic Properties of $\mathrm{Pt} / \mathrm{Co} / \mathrm{Pt}$ Films by $\mathrm{Ga}+$ Ion Irradiation: Focused versus Uniform Irradiation," Acta Physica Polonica A, Feb. 2018. [Online]. Available: https://hal.archives-ouvertes.fr/hal-02331893

[31] J. Ferré, C. Chappert, H. Bernas, J. P. Jamet, P. Meyer, O. Kaitasov, S. Lemerle, V. Mathet, F. Rousseaux, and H. Launois, "Irradiation induced effects on magnetic properties of $\mathrm{Pt} / \mathrm{Co} / \mathrm{Pt}$ ultrathin films," Journal of Magnetism and Magnetic Materials, vol. 198, no. 1-3, pp. 191-193, Jun. 1999.

[32] B. M. S. Teixeira, A. A. Timopheev, N. Caçoilo, S. Auffret, R. C. Sousa, B. Dieny, and N. A. Sobolev, "Stabilization of the easy-cone magnetic state in free layers of magnetic tunnel junctions," Phys. Rev. B, vol. 100, p. 184405, Nov 2019. [Online]. Available: https://link.aps.org/doi/10.1103/PhysRevB.100.184405

[33] K.-M. Lee, J. Choi, J. Sok, and B.-C. Min, "Temperature dependence of the interfacial magnetic anisotropy in w/cofeb/mgo," AIP Advances, vol. 7, p. 065107, 062017.

[34] J.-Y. Park, J.-M. Kim, J. Ryu, J. Jeong, and B.-G. Park, "Effects of proton and ion beam radiation on magnetic tunnel junctions," Thin Solid Films, vol. 686, p. 137432, Sep. 2019.

[35] A. Debelle, M. Backman, L. Thomé, W. J. Weber, M. Toulemonde, S. Mylonas, A. Boulle, O. H. Pakarinen, N. Juslin, F. Djurabekova, K. Nordlund, F. Garrido, and D. Chaussende, "Combined experimental and computational study of the recrystallization process induced by electronic interactions of swift heavy ions with silicon carbide crystals," Phys. Rev. B, vol. 86, p. 100102, Sep 2012. [Online]. Available: https://link.aps.org/doi/10.1103/PhysRevB.86.100102 